INT. J. CONTROL, 1989 , vOL. 49 , NO. 1, 161-168

\title{
Set of open-loop block-diagonalizers of transfer matrices
}

\author{
A. BÜLENT'ÖZGÜLER $†$ and VASFİ ELDEM $\ddagger$
}

The set of open-loop (right) block-diagonalizers of a given $p \times m$ transfer matrix $Z$, with specified block sizes, is studied. A characterization of this set is obtained in terms of a 'maximal' open-loop block-diagonalizer and a minimal proper basis of the kernel of $Z$. The relevance of the results to some non-interacting control problems is pointed out. In particular, the results obtained yield a minimum delay solution to the block-decoupling problem using dynamic state feedback.

\section{Introduction}

In this paper, we present a systematic approach to the study of the set of open-loop block-diagonalizers of a transfer matrix. We characterize (Theorems 1 and 2) all elements of this set via a representative of the subset of 'maximal open-loop diagonalizers' and a proper basis matrix for the kernel of the transfer matrix. The special open-loop diagonalizers defined via the Hermite normal form have the property of having a 'minimum rank zero coefficient matrix' (Corollary 1). These results lead to an easy recovery and extension (Theorem 3 ) of certain results that find application in non-interacting control. In particular, the recent result of Dion and Commault (1985) on the diagonalization problem by dynamic state feedback is easily extended to the block-diagonal case (Corollary 2).

In $\S 2$, we examine the simpler problem of diagonalization in detail. These results are then extended to block-diagonalization in $\S 3$, where the treatment is quite concise since the details parallel the development of $\S 2$.

A short description of the terminology and notation used in the paper is as follows. We define the (causality) degree of a rational function of $z$ with real coefficients (i.e. an element of the field of real rational functions $\mathbb{R}(z)$ ) to be the difference between the degrees of its numerator and denominator polynomials. Thus, if $a$ is in $\mathbb{R}(z)$, then $a=p / q$ for (coprime) polynomials $p$ and $q$ in $\mathbb{R}[z]$ and $\operatorname{deg} a:=\operatorname{deg} p-\operatorname{deg} q$. The degree of 0 is assigned to be $-\infty$ for convenience. An element $a$ in $\mathbb{R}(z)$ is called proper if and only if deg $a \leqslant 0$ and strictly proper if and only if deg $a<0$. The set of proper elements of $\mathbb{R}(z)$ form the ring of transfer functions (Morse 1975), denoted by $\mathbb{R}_{\text {pr }}(z)$. A degree 0 element (unit) of this ring is said to be biproper.

If $A$ is in $\mathbb{R}(z)^{p \times m}$, then $\operatorname{deg} A:=\max \left\{\operatorname{deg} A_{i j}\right\}$, for $i=1, \ldots, p$ and $j=1, \ldots, m$. A rational matrix is proper if and only if $\operatorname{deg} A \leqslant 0$ and strictly proper if and only if $\operatorname{deg} A<0$. A $p \times m$ rational matrix is called left biproper if and only if it is proper and admits a proper right inverse, i.e. there exists a proper $m \times p$ matrix $B$ such that $A B=I$; it is called right biproper if and only if its transpose $A^{\prime}$ is left biproper. If a square rational matrix is left (or right) biproper, then it is called biproper. If $A_{0}$

Received 29 February 1988. Revised 15 April 1988.

$\dagger$ Electrical and Electronics Engineering, Bilkent University, P.O. Box 8, Maltepe 06572, Ankara, Turkey.

$\ddagger$ Research Institute for Basic Sciences, Tübitak, P.O. Box 74, Gebze 41470, Kocaeli, Turkey.

0020-7179/89 \$3.00 @ 1989 Taylor \& Francis Ltd. 
denotes the coefficient of $z^{0}$ in the Laurent series expansion in $z^{-1}$ of $A$, then a proper $A$ is well known to be left biproper if and only if rank $A_{0}=p$, or equivalently, $A_{0}$ is left invertible. Finally, we define the concept of row properness for a rational matrix. Let $A$ be in $\mathbb{R}(z)^{p \times m}$ with $A_{i}$ denoting its $i$ th row, a $1 \times m$ matrix. Let $\mu_{i}:=\operatorname{deg} A_{i}$ and define the row degree matrix $D$ of $A$ to be $D:=\operatorname{diag}\left\{z^{\mu_{i}}\right\}$ which is a diagonal nonsingular matrix. Let $B:=D^{-1} A$. The rational matrix $A$ is called row proper if and only if $B$ is left biproper. The zero coefficient matrix $B_{0}$ of the proper matrix $B$ is called the highest row coefficient matrix of $A$ and is denoted by $A_{h}$. We note that if $A$ is a polynomial matrix, then our concept of row properness is precisely that of Wolovich (1974). It is easy to see that any rational $A$ (row proper or not) can be uniquely represented as $A=D\left(A_{h}+Y\right)$ for some strictly proper rational matrix $Y$. (The dual concepts are defined similarly or via 'matrix transpose'.)

\section{Open-loop diagonalizers}

Let $Z$ be a $p \times m$ transfer matrix. Another $m \times p$ transfer matrix $T$ is called an open loop diagonalizer (OLD) of $Z$ if and only if $D:=Z T$ is diagonal and non-singular. In this section, the set of OLDs of $Z$ are examined. A convenient characterization of this set is obtained and certain elements of special properties relevant to diagonalization problems are identified.

We start by observing that the condition 'rank $Z=p$ ' over $\mathbb{R}(z)$ is necessary for the existence of an OLD of $Z$ since $D$ is required to be non-singular. This condition is also sufficient, i.e. any full row-rank $Z$ has an OLD. We prove this via the Hermite normal form (Morse 1975) of $Z$ over $\mathbb{R}_{p r}(z)$ under right equivalence. For convenience, we fix a complete residue system and a complete system of non-associates with respect to the monic polynomial $z$. Then, there exists a biproper matrix $B$ such that $Z B=\left[\begin{array}{ll}H & 0\end{array}\right]$, where $H=D U$ for some unimodular polynomial matrix $U$ and a proper diagonal matrix $D=\operatorname{diag}\left\{z^{-d_{i}}\right\}$ with non-negative integers $d_{i}(i=1, \ldots, p)$. Consequently, $H^{-1}=U^{-1} D^{-1}$ is a polynomial matrix of column degrees, say, $e_{i}(i=1, \ldots, p)$. If we define

$$
T_{m}=B_{1} H^{-1} D_{m}
$$

where $B_{1}$ is the submatrix consisting of the first $p$ columns of $B$ and $D_{m}:=\operatorname{diag}\left\{z^{-e_{i}}\right\}$, then $Z T_{m}=D_{m}$ with $D_{m}$ non-singular and $T_{m}$ proper. Note that the condition 'rank $Z=p$ ' is used to assure that $H^{-1}$ exists. The integers $e_{i}(i=1, \ldots, p)$ are called the essential orders of $Z$ by Commault and Dion (1986) and Commault et al. (1986). Since $H$ is unique, it follows that the essential orders associated with all OLDs of $Z$, defined via the Hermite normal form of $Z$ as above, are the same.

Let $W_{m}$ be a right biproper matrix, the columns of which constitute a basis of ker $Z$. If rank $Z=p$, then $W_{m}$ has size $m \times(m-p)$ and $B_{2}$ defined by $B=\left[\begin{array}{ll}B_{1} & B_{2}\end{array}\right]$ of the construction above is one such basis matrix. A useful property of $T_{m}$ of (1) is that

$$
\operatorname{Im} T_{m 0} \cap \operatorname{Im} W_{m 0}=0
$$

In fact, from (1) and the properness of $H^{-1} D_{m}, \operatorname{Im} T_{m 0}$ is in $\operatorname{Im} B_{10}$ and, from the biproperness of $B, \operatorname{Im} B_{10} \cap \operatorname{Im} B_{20}=0$; implying that $\operatorname{Im} T_{m 0} \cap \operatorname{Im} B_{20}=0$. However, any basis matrix $W_{m}$ of $Z$ is related to $B_{2}$ by $W_{m}=B_{2} A$ for some biproper $A$. It follows that $\operatorname{Im} W_{m 0}=\operatorname{Im} B_{20}$, yielding (2). Also, observe that each column of $T_{m}$ of (1) is right biproper, i.e. $\operatorname{deg} T_{m i}=0$ for all $i=1, \ldots, p$. We now show that any $T_{m}$ defined through the Hermite normal form as above achieves the maximal column degrees for $D=Z T$. 


\section{Lemma 1}

If $T$ is an OLD of $Z$ with $D=Z T$ then $D_{m}^{-1} D$ is proper.

Proof

The proof is by contradiction. Let $E:=\mathrm{D}_{m}^{-1} D$ and suppose deg $E_{i i}>0$ for some $i$. Then the $i$ th column $T_{i}$ of $T$ satisfies $Z T_{i} E_{i i}^{-1}=Z T_{m i}=D_{m i i}$ and hence the vector $T_{i} E_{i i}^{-1}-T_{m i}$ is in ker $Z$, i.e. $T_{i} E_{i i}^{-1}-T_{m i}=W_{m} y$ for some proper vector $y$, and is itself proper. Furthermore, as $T_{i} E_{i i}^{-1}$ is strictly proper by deg $E_{i i}>0$, we have $\left(T_{i} E_{i i}^{-1}\right.$ $\left.-T_{m i}\right)_{0}=T_{m i 0}$. Therefore, $W_{m 0} y_{0}=T_{i m 0}$ and this contradicts (2) since $T_{m i 0}$ is non-zero.

A characterization of the set of all OLDs of $Z$ may now be given in terms of $T_{m}$ and $W_{m}$.

\section{Theorem 1}

Let rank $Z=p$ and let $T_{m}$ be defined by (1). Also let $W_{m}$ be a right biproper basis matrix of ker $Z$. An $m \times p$ transfer matrix $T$ is an OLD of $Z$ if and only if

$$
T=T_{m} Y_{d}+W_{m} Y
$$

for some transfer matrices $Y_{d}$ and $Y$, with $Y_{d}$ diagonal and non-singular.

\section{Proof}

Clearly, any $T$ defined by (3) for some $Y_{d}$ and $Y$ with stated properties is proper and satisfies $Z T=D_{m} Y_{d}$. Hence, it is an OLD of $Z$ achieving $D=D_{m} Y_{d}$. Conversely, if $T$ is an OLD of $Z$ so that $D=Z T$ is diagonal and non-singular then from (1), $T-T_{m} D_{m}^{-1} D=W_{m} Y$ for some $(m-p) \times p$ rational matrix $Y$. Since $Y_{d}:=D_{m}^{-1} D$ is proper by Lemma 1 and since $W_{m}$ is right biproper, it follows that $Y$ is proper. (Actually, $Y=W\left(T-T_{m} Y_{d}\right)$ for a proper left inverse $W$ of $W_{m}$.) Consequently, (3) holds with $Y_{d}$ proper, diagonal and non-singular, and with $Y$ proper.

Let an OLD $T_{M}$ of $Z$ be called maximal if and only if $D_{M}^{-1} D_{m}$ is biproper, where $D_{M}:=Z T_{M}$, i.e. if and only if it achieves the same orders as $T_{m}$ of (1). An easy consequence of Theorem 1 is that an OLD $T_{M}$ of $Z$ is maximal if and only if

$$
T_{M}=T_{m} B_{d}+W_{m} Y
$$

for some biproper diagonal $B_{d}$ and proper $Y$. We now show that a maximal OLD has all its columns right biproper and that in the set of maximal OLDs of $Z$ the elements defined via the Hermite normal form of $Z$ have 'minimal' zero-coefficient matrices.

\section{Corollary 1}

If $T_{M}$ is a maximal OLD of $Z$, then

(i) $\operatorname{deg} T_{M i}=0$ for all $i=1, \ldots, p$

(ii) rank $T_{M 0} \geqslant$ rank $T_{m 0}$

(iii) if $T_{m}^{1}$ and $T_{m}^{2}$ are two maximal OLDs defined via the Hermite normal form of $Z$ as in (1), then

$$
\operatorname{rank} T_{m 0}^{1}=\operatorname{rank} T_{m 0}^{2}
$$


Proof

Let $T_{M}$ be written as in (4) for some $B_{d}$ and $Y$. If (i) fails, then for some $i$ we must have deg $T_{M i}<0$. This means $T_{M i 0}=0$ which implies, from (4), that $T_{m i 0}$ is in Im $W_{m 0}$, contradicting (2). This establishes (i). If (ii) fails, then rank $T_{M 0}<$ rank $T_{m 0}=$ rank $T_{m 0} B_{d 0}$, where the equality is by non-singularity of $B_{d 0}$. Hence, there exists a non-zero constant vector $\alpha$ such that $T_{M 0} \alpha=0$ whereas $T_{m 0} B_{d 0} \alpha$ is non-zero. Using (4), we have $T_{M 0}=T_{m 0} B_{d 0}+W_{m 0} Y_{0}$ which implies that $T_{m 0} B_{d 0} \alpha-W_{m 0} Y_{0} \alpha=0$ or that (2) fails to hold. This establishes (ii). Finally, statement (iii) follows directly from (ii), since in this case the inequality works both ways.

\section{Remark}

Note that (i) does not necessarily imply that $T_{M}$ is maximal and also note that rank equality need not hold in (ii). In fact, consider the transfer matrix $Z$ for which one particular $T_{m}$ computed by (1) turns out to be as below

$$
Z=\left[\begin{array}{ccc}
1 & 1 & z^{-1} \\
1 & 1 & -z^{-1}
\end{array}\right], \quad T_{m}=\left[\begin{array}{cc}
0 & 0 \\
z^{-1} & z^{-1} \\
1 & -1
\end{array}\right]
$$

The following OLD $T$ of $Z$ has $\operatorname{deg} T_{i}=0$, for $i=1,2$, but it is not maximal and $T_{M}$ below is a maximal OLD of $Z$ but rank $T_{M 0}=2>1=\operatorname{rank} T_{m 0}$

$$
T=\left[\begin{array}{cc}
0 & 1 \\
z^{-1} & -1+z^{-2} \\
1 & -z^{-1}
\end{array}\right], \quad T_{M}=\left[\begin{array}{cc}
0 & 1 \\
z^{-1} & -1+z^{-1} \\
1 & -1
\end{array}\right]
$$

\section{Block-diagonalizers}

Let us now consider a more general problem of open-loop diagonalization for a $p \times m$ transfer matrix $Z$. We call an $m \times p$ transfer matrix $T$ an open-loop blockdiagonalizer (OLBD) of $Z$ with block sizes $\left\{p_{i}\right\}\left(\left\{p_{i}\right\}\right.$-OLBD) if and only if $D:=Z T$, where $D=\operatorname{diag}\left\{D_{1}, \ldots, D_{k}\right\}$ with $D_{i}$ a $p_{i} \times p_{i}$ non-singular matrix for $i=1, \ldots, k$. Clearly, it must hold that

$$
p_{1}+\cdots+p_{k}=p
$$

From the non-singularity of $D$, the condition 'rank $Z=p$ ' over $\mathbb{R}(z)$ is again necessary for the existence of a $\left\{p_{i}\right\}$-OLBD. We now generalize the procedure of the previous section to construct a 'maximal OLBD' for a full row-rank transfer matrix $Z$.

Let $Z B=\left[\begin{array}{ll}H & 0\end{array}\right]$, where $H$ is the Hermite normal form of $Z$ and $B$ is a biproper matrix, as before. We partition the columns of the polynomial matrix $\hat{H}:=H^{-1}$ as

$$
\hat{H}=\left[\begin{array}{lll}
\hat{H}_{1} & \ldots & \hat{H}_{k}
\end{array}\right]
$$

where $\hat{H}_{i}$ has $p_{i}$ columns. Let $P_{i}$ be a minimal polynomial basis matrix for $\operatorname{Im} \hat{H}_{i}$ which is an $\mathbb{R}(z)$-vector space with dimension $p_{i}$ (see Forney 1975). Thus, $P_{i}$ is an $m \times p_{i}$ right unimodular and column proper polynomial matrix; and for some non-singular polynomial matrix $G_{i}$, we have

$$
P_{i} G_{i}=\hat{H}_{i}
$$


From the column properness of $P_{i}$, its highest column coefficient matrix $P_{i h}$ has full column rank, implying the existence of a $p_{i} \times p_{i}$ minor $M_{i}$ of $P_{i}$ such that

$$
P_{i} M_{i}^{-1} \text { is right biproper }
$$

(Note that the matrix $M_{i}$ can be chosen by picking indexes $j_{1}, \ldots, j_{p_{i}}$ such that the corresponding rows of $P_{i h}$ are linearly independent and then forming $M_{i}$ out of the corresponding rows of $P_{i}$.) We now prove the following result as a first step in defining a maximal $\left\{p_{i}\right\}$-OLBD of $Z$.

\section{Lemma 2}

The matrices $D_{i}^{*}:=\left(M_{i} G_{i}\right)^{-1}$ and $\hat{H}_{i} D_{i}^{*}$ are proper, and given any other proper matrix $E_{i}$ such that $\hat{H}_{i} E_{i}$ is proper, $\left(D_{i}^{*}\right)^{-1} E_{i}$ is proper.

Proof

By the definition of $D_{i}^{*}$, we have $P_{i} M_{i}^{-1}=\hat{H}_{i} D_{i}^{*}$. Now, $H_{i} \hat{H}_{i}=I$ where $H_{i}$ denotes the $i$ th block row of the matrix $H$. It follows that $D_{i}^{*}=H_{i} P_{i} M_{i}^{-1}$ and $\hat{H}_{i} D_{i}^{*}$ are both proper by the properness of $H_{i}$ and $P_{i} M_{i}^{-1}$. This proves the first part of the claim. Now suppose $Y_{i}:=\hat{H}_{i} E_{i}$ is proper. We have

$$
Y_{i} E_{i}^{-1}-P_{i} M_{i}^{-1}\left(D_{i}^{*}\right)^{-1}=0
$$

This implies, by the biproperness of $P_{i} M_{i}^{-1}$ that $\left(D_{i}^{*}\right)^{-1} E_{i}$ is proper.

We now define

$$
T^{*}:=B_{1} \hat{H} D^{*} \quad \text { with } D^{*}:=\operatorname{diag}\left\{D_{1}^{*}, \ldots, D_{k}^{*}\right\}
$$

and observe that $T^{*}$ is proper and

$$
Z T^{*}=D^{*}
$$

Moreover, if, for some $\left\{p_{i}\right\}$-OLBD $T$ of $Z$, one has $Z T=E$ for some non-singular $E=\operatorname{diag}\left\{E_{1}, \ldots, E_{k}\right\}$, then $\left[\begin{array}{ll}H & 0\end{array}\right] B^{-1} T=E$ implying that $\hat{H}_{i} E_{i}$ is proper for $i=1$, $\ldots, k$. By Lemma 2 , this implies that $\left(D_{i}^{*}\right)^{-1} E_{i}$, and hence $\left(D^{*}\right)^{-1} E$, is proper. Therefore, $T^{*}$ is a maximal $\left\{p_{i}\right\}$-OLBD of $Z$.

In the same manner as Theorem 1, we can prove the following characterization of the set of all $\left\{p_{i}\right\}$-OLBDs of $Z$.

\section{Theorem 2}

Let $W_{m}$ be a right biproper basis matrix of $\operatorname{ker} Z$ and let $T^{*}$ be defined by (5). An $m \times p$ transfer matrix $T$ is a $\left\{p_{i}\right\}$-OLBD of $Z$ if and only if

$$
T=T^{*} Y_{b d}+W_{m} Y
$$

for some $\left\{p_{i}\right\}$-block diagonal proper $Y_{b d}$ and proper $Y$.

If the matrix $Y_{b d}$ of (6) is biproper then clearly the set of matrices $T$ obtained by (6) yields the set of all maximal $\left\{p_{i}\right\}$-OLBDs of $Z$. Let $Z T_{M}^{*}=D_{M}^{*}$ for a maximal $\left\{p_{i}\right\}$ OLBD $T_{M}^{*}$ of $Z$. From (6), $\left(D_{M}^{*}\right)^{-1} D^{*}$ is biproper. Therefore, all maximal $\left\{p_{i}\right\}$ OLBDs achieve the same row degree set as that of $D^{*}$. Let us denote

$$
e_{i}^{*}:=-\left(i \text { th row degree of } D^{*}\right), \quad i=1, \ldots, p
$$


These non-negative integers serve as natural generalizations of the essential orders $\left\{e_{i}\right\}$ of $\S 2$ and it can easily be shown, similar to Commault et al. $(1986, \S 4)$, that they are invariant under right multiplication of $Z$ by biproper matrices. Since $D_{m}$ of $\S 2$ is a $\left\{p_{i}\right\}$-OLBD of $Z$ for any choice of the set $\left\{p_{i}\right\}$, it follows from the maximality of $D^{*}$ that

$$
e_{i}^{*} \leqslant e_{i}, \quad i=1, \ldots, p
$$

Observe that, similar to $T_{m}$ of the previous section, $T^{*}$ satisfies

$$
\operatorname{Im} T_{0}^{*} \cap \operatorname{Im} W_{m 0}=0
$$

Using this property, it can easily be shown that for a maximal $\left\{p_{i}\right\}$-OLBD $T_{M}$ of $Z$, the conditions (ii) and (iii) of Corollary 1 still hold on replacing $T_{m}$ by $T^{*}$. (Condition (i) also holds but it can be strengthened to read: 'The $i$ th column block $\left(T_{M}\right)_{i}$ of $T_{M}$ is right biproper for $i=1, \ldots, k^{\prime}$.)

As an important consequence of the characterizations above, we now prove the following results. For this, we need a natural extension of the concept of row properness to block-rows.

\section{Definition}

A $p \times m$ transfer matrix $Z$ is called $\left\{p_{i}\right\}$-block-row proper if and only if there exists a non-singular $\left\{p_{i}\right\}$-block diagonal matrix $D$ and a left biproper matrix $B$ such that $Z=D B$.

Note that for $p_{i}=1, i=1, \ldots, p$, this definition coincides with row properness. In the other extreme case of $k=1$ and $p_{1}=p, Z$ is $\left\{p_{i}\right\}$-block-row proper if and only if rank $Z=p$.

\section{Theorem 3}

Consider a $p \times m$ transfer matrix $Z$ with rank $Z=p$.

(i) $Z$ has a right biproper $\left\{p_{i}\right\}$-OLBD if and only if rank $T_{0}^{*} \geqslant 2 p-m$, in which case it also admits a maximal right biproper $\left\{p_{i}\right\}$-OLBD.

(ii) There exists a $\left\{p_{i}\right\}$-OLBD $T$ of $Z$ such that $Z T=D$ with $D^{-1} Z$ left biproper if and only if $Z$ is $\left\{p_{i}\right\}$-block-row proper. Such $\left\{p_{i}\right\}$-OLBDs are maximal and right biproper.

\section{Proof}

(i) Let $T$ be a right biproper $\left\{p_{i}\right\}$-OLBD of $Z$. By Theorem $1, T=T^{*} Y_{b d}+W_{m} Y$ for some proper, block-diagonal, and non-singular $Y_{b d}$ and a proper $Y$. Thus, $T_{0}=T_{0}^{*} Y_{b d 0}+W_{m 0} Y_{0}$ which implies as rank $T_{0}=p$ that $\operatorname{rank}\left[\begin{array}{cc}T_{0}^{*} & W_{m 0}\end{array}\right] \geqslant p$. Since rank $W_{m 0}=m-p$ and since $\operatorname{Im} T_{0}^{*} \cap \operatorname{Im} W_{m 0}=0$, we must have rank $T_{m 0} \geqslant 2 p-m$. Conversely, if rank $T_{0}^{*} \geqslant 2 p-m$, then by (6), we have $\operatorname{rank}\left[\begin{array}{cc}T_{0}^{*} & W_{m 0}\end{array}\right] \geqslant p$. We now construct a right biproper $\left\{p_{i}\right\}$-OLBD of $Z$. Let columns $\left\{j_{1}, \ldots, j_{2 p-m}\right\}$ of $T_{0}^{*}$ be linearly independent. Now define an $(m-p) \times p$ constant matrix $K$ by $K_{i i}=1$ if $i$ is not in the set $\mathbf{s}:=\left\{j_{1}, \ldots, j_{2 p-m}\right\}$ and $K_{i j}=0$ otherwise. Consider

$$
T=T^{*}+W_{m} K
$$

which is clearly a maximal OLBD. Furthermore, it is right biproper as we now prove. 
Suppose rank $T_{0}<p$ so that for some constant vector $\alpha$, we have $T_{0} \alpha=T_{0}^{*} \alpha+W_{m 0} K \alpha$ $=0$. This implies from (7) that $T_{0}^{*} \alpha=0$ and $W_{m 0} K \alpha=0$. By the fact that $W_{m 0}$ is of full column rank, the second equality yields $K \alpha=0$. By definition of $K$, we have $\alpha_{i}=0$ for all $i$ not in $\mathbf{s}$ due to $K \alpha=0$ and for all $i$ in $s$ due to $T_{0}^{*} \alpha=0$. Thus, $\alpha=0$. This proves that $T$ is right biproper.

(ii) Let $Z$ be $\left\{p_{i}\right\}$-block-row proper so that $Z=D B$ for a $\left\{p_{i}\right\}$-block diagonal $D$, where $B T=I$ for some proper $T$ from the left biproperness of $B$. Hence, $Z T=D$ with $T$ right biproper. Conversely, if $Z T=D$ for some $\left\{p_{i}\right\}$-OLBD of $Z$ such that $B:=D^{-1} Z$ is proper, then directly by definition, $Z$ is $\left\{p_{i}\right\}$-block-row proper. As $D^{-1} D^{*}=D^{-1} Z T^{*}$ is proper, we also have that $T$ is maximal. Furthermore, $B T=I$ implying that $T$ is right biproper.

The second part (ii), is a generalization of the following result of Verghese and Kailath (1981) and Ohta and Kodama (1985), concerning row properness. There exists an OLD of $Z$ with $\operatorname{deg} D_{i i}=i$ th row degree of $Z$ if and only if $Z$ is row proper. This specialization to the case $p=k$ of Theorem 3 (ii) implies the following fact concerning the relation between essential orders and the 'generalized' essential orders $\left\{e_{i}^{*} ; i=1, \ldots, p\right\}$. If $Z$ is row proper, then the equality holds in (7) for all partitioning $\left\{p_{i}\right\}$ of the rows of $Z$. This is because, if $Z$ is row proper, then $e_{i}$ is the $i$ th row degree of $Z$ and $Z=D_{m} B$ for a left biproper $B$. Thus, $D_{m}^{-1} D^{*}=B T^{*}$ is proper; yielding equality in (7).

The first part (i) of Theorem 3, when coupled with Theorem 3 of Commault and Dion (1986), yields a 'minimum delay decoupled' solution to block-decoupling by dynamic state feedback problem with specified block sizes (see Dion and Commault 1985, Commault and Dion 1986) for a strictly proper transfer matrix $Z$ with 'null static kernel', i.e. with ker $Z \cap \mathbb{R}^{m}=\{0\}$. We give a formal definition of the problem for convenience.

\section{Problem definition}

Given an $n$-dimensional realization $\Sigma_{Z}=(A, B, C)$ of $Z$, determine a constant $m \times p$ matrix $L$ and an $m \times n$ transfer matrix $F$ such that the closed-loop transfer matrix

$$
\hat{Z}=C(z I-A-B F)^{-1} B L
$$

is non-singular and $\left\{p_{i}\right\}$-block diagonal.

A solution to this problem in the special case of $p=k$ and $p_{i}=1$, i.e. to the diagonalization problem by dynamic state feedback, was given by Dion and Commault (1985). The proof below, when specialized to the case $p=k$ and $p_{i}=1$, may be viewed as an alternative and shorter proof to that of Dion and Commault (1985).

\section{Corollary 2}

Let $Z$ be a $p \times m$ full row rank, strictly proper transfer matrix of null static kernel. The block decoupling problem by dynamic state feedback (and constant precompensator) is solvable for any realization $\Sigma_{Z}=(A, B, C)$ of $Z$ if and only if

$$
\text { rank } T_{0}^{*} \geqslant 2 p-m
$$

in which case, there also exists a minimum delay decoupled solution. 
Proof

Only if. Let a pair $(L, F)$ with the stated properties exist so that $\hat{Z}$ is $\left\{p_{i}\right\}$-block diagonal. Then, $T:=\left[I-F(z I-A)^{-1} B\right]^{-1} L$ is easily seen to be a right biproper $\left\{p_{i}\right\}-$ OLBD of $Z$-since $T_{0}=L$, where the latter is of full column rank by non-singularity of $\hat{Z}$. From Theorem 3 (i), it follows that the rank inequality holds.

If. By the hypothesis of 'null static kernel', the matrix $B$ is of full column rank. Let $B^{*}$ be such that $B^{*} B=I$. If the rank inequality holds, then Theorem 3 (i) implies the existence of a maximal right biproper $\left\{p_{i}\right\}$-OLBD, say $T_{M}$, of $Z$. Let $T_{M 0}^{*}$ be such that $T_{M 0}^{*} T_{M 0}=I$, which exists by right biproperness of $T_{M}$. We now define

$$
L:=T_{M 0}, \quad F:=\left(I-T_{M} T_{M 0}^{*}\right) B^{*}(z I-A)
$$

and note that $F$ is proper since $I-T_{M} T_{M O}^{*}$ is strictly proper. Moreover

$$
\hat{Z}=Z\left[I-F(z I-A)^{-1} B\right]^{-1} L=Z T_{M}=D^{*} B_{b d}
$$

for some biproper and $\left\{p_{i}\right\}$-block diagonal $B_{b d}$, where the last equality is given by the fact that $T_{M}$ is 'maximal'. This establishes that the constructed pair $(L, F)$ is a solution to the problem and that they achieve maximum possible causality degrees $\left\{e_{i}^{*} ; i=1, \ldots, p\right\}$.

\section{Conclusions}

We have obtained a useful characterization, in Theorems 1 and 2, of the set of all open-loop block-diagonalizers (decouplers) of a given transfer matrix. An essential element of this description has been a maximal $\left\{p_{i}\right\}-O L B D\left(T^{*}\right)$ defined naturally via the Hermite normal form over $\mathbb{R}_{p r}(z)$ of the transfer matrix. We have also obtained suitable extensions of the concepts of row properness and essential orders for use in block decoupling problems.

This characterization is bound to find many applications in feedback-decoupling theory in view of the fact that most of the feedback-decoupling problems can be formulated as open-loop problems after suitable transformations. Block-decoupling by dynamic state feedback is one such problem and we have obtained a solution, in Corollary 2, of this problem by the use of Theorem 2. A further application of the characterization result of this paper is in the diagonalization by dynamic output feedback problem considered by Eldem and Özgüler (1987), where Theorem 1 is used to describe the set of all solutions.

\section{REFERENCES}

Commault, C., Descusse, J., Dion, J. M., Lafay, J. F., and Malabre, M., 1986, Int. J. Control, $44,689$.

Commault, C., and Dion, J. M., 1986, Some structural invariants within the transfer matrix approach. Proc. 25th I.E.E.E. Conf. on Decision and Control, Athens, pp. 1284-1288.

Dion, J. M., and Commault, C., 1985, On linear dynamic state feedback decoupling. Proc. 24th I.E.E.E. Conf. on Decision and Control, Fort Lauderdale, pp. 183-188.

ELDEM, V., and ÖZGÜLER, A. B., 1987. A solution to diagonalization problem by constant precompensator and dynamic output feedback. I.E.E.E. Trans. autom. Control, to be published.

ForNeY, G. D., JR., 1975, SIAM J. Control Optim., 13, 493.

MORSE, A. S., 1975, System invariants under feedback and cascade control, in Lecture Notes in Economics and Mathematical Systems, Vol. 131 (New York: Springer).

OHTA, Y., and Kodama, S., 1985, I.E.E.E. Trans. autom. Control, 30, 818.

Verghese, G. C., and Kallath, T., 1981, I.E.E.E. Trans. autom. Control, 26, 434.

Wolovich, W. A., 1974, Linear Multivariable Systems (New York: Springer-Verlag). 